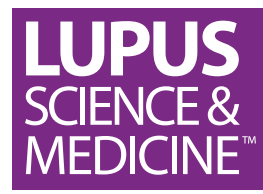

Fishbein J, Hong J, et al. Quinolinic acid, a kynurenine/ tryptophan pathway metabolite, associates with impaired cognitive test performance in systemic lupus erythematosus. Lupus Science \& Medicine 2021;8:e000559. doi:10.1136/ lupus-2021-000559

- Additional supplemental material is published online only. To view, please visit the journal online (http://dx.doi.org/10. 1136/lupus-2021-000559).

This work was previously presented as a poster at the American College of Rheumatology Convergence 2020 (Anderson E Fishbein $\mathrm{J}$, Hong J, et al. Impact of the Kynurenine/Tryptophan Pathway on Cognitive Dysfunction and Depression in Systemic Lupus Erythematosus (abstract). Arthritis Rheumatol. 2020;72(suppl10)).

Received 12 August 2021 Accepted 12 October 2021

Check for updates

(C) Author(s) (or their employer(s)) 2021. Re-use permitted under CC BY-NC. No commercial re-use. See rights and permissions. Published by BMJ.

For numbered affiliations see end of article.

Correspondence to

Dr Erik W Anderson;

eanderson22@northwell.edu

\title{
Quinolinic acid, a kynurenine/ tryptophan pathway metabolite, associates with impaired cognitive test performance in systemic lupus erythematosus
}

\author{
Erik W Anderson (D) , ${ }^{1}$ Joanna Fishbein, ${ }^{1}$ Joseph Hong, ${ }^{1}$ Julien Roeser, ${ }^{2}$ \\ Richard A Furie (D) , 'Cynthia Aranow (D) , ${ }^{1}$ Bruce T Volpe, ${ }^{1}$ Betty Diamond (D) , \\ Meggan Mackay ${ }^{1}$
}

\section{ABSTRACT}

Objectives Interferon-alpha, an important contributor to SLE pathogenesis, induces the enzyme indoleamine 2,3-dioxygenase in the kynurenine/tryptophan (KYN/TRP) pathway. This leads to a potentially neurotoxic imbalance in the KYN/TRP pathway metabolites, quinolinic acid (QA), an N-methyl D-aspartate glutamatergic receptor (NMDAR) agonist, and kynurenic acid (KA), an NMDAR antagonist. We determined whether QA/KA ratios associate with cognitive dysfunction (CD) and depression in SLE.

Methods This cross-sectional study included 74 subjects with SLE and 74 healthy control (HC) subjects; all without history of neuropsychiatric disorders. Serum metabolite levels (KYN, TRP, QA, KA) were measured concurrently with assessments of cognition (Automated Neuropsychological Assessment Metrics (ANAM), $2 \times 2$ array), mood and pain, and compared between SLE and HC. Multivariable modelling in SLE was used to evaluate associations of metabolites with cognitive performance and depression.

Results Serum KYN/TRP and QA/KA ratios were elevated in SLE versus $H C(p<0.0001)$. SLE performed worse than $\mathrm{HC}$ on four of five ANAM tests (all $p \leq 0.02$ ) and the $2 \times 2$ array $(p<0.01)$, and had higher depression scores $(p<0.01)$. In SLE, elevated $Q A / K A$ ratios correlated with poor performance on Match to Sample (MTS), a working memory and visuospatial processing task $(p<0.05)$. Subjects with SLE with elevated QA/KA ratios also had slightly higher odds of depression, but this did not reach significance $(\mathrm{p}=0.09)$. Multivariable modelling in SLE confirmed an association between QA $\mathrm{KA}$ ratios and poor MTS performance when considering potentially confounding factors $(p<0.05)$.

Conclusions Elevated serum KYN/TRP and QA/KA ratios confirm KYN/TRP pathway activation in SLE. The novel association between increased QA/KA ratios and poor cognitive performance supports further study of this pathway as a potential biomarker or therapeutic target for SLEmediated CD.

\section{INTRODUCTION}

Cognitive dysfunction (CD) is an insidious, highly prevalent and often devastating

\section{Key messages}

What is already known about this subject?

- Interferon-alpha contributes to lupus pathogenesis and induces the enzyme indoleamine 2,3-dioxygenase in the kynurenine/tryptophan (KYN/ TRP) pathway, which leads to a potentially neurotoxic imbalance of elevated quinolinic acid $(Q A)$ relative to kynurenic acid (KA).

- An elevated QA/KA ratio associates with cognitive dysfunction in neurodegenerative diseases and idiopathic depression.

What does this study add?

- It was not known whether elevated QA/KA ratios were present in lupus, and whether they associated with cognitive dysfunction or depression in patients with lupus. In this study, elevated QA/KA ratios in patients with lupus associate with cognitive dysfunction; namely, poor performance in working memory and visuospatial processing.

How might this impact on clinical practice or future developments?

- The association of elevated QA/KA ratios with cognitive dysfunction in patients with lupus, supported by a plausible pathophysiological mechanism of interferon-alpha stimulation of the KYN/TRP pathway, suggests a potential therapeutic target that deserves future study.

manifestation of SLE with significant impact on quality of life. ${ }^{1}$ Abnormal brain imaging and evidence of $\mathrm{CD}$ in patients with newonset disease suggest that SLE-related mechanisms contribute to $\mathrm{CD}$ irrespective of other potentially neurotoxic processes such as infection, comorbid illness and medications. ${ }^{23}$ Several mechanisms for SLE-mediated CD (SLE-CD) have been identified in animal models, including pathways mediated by 


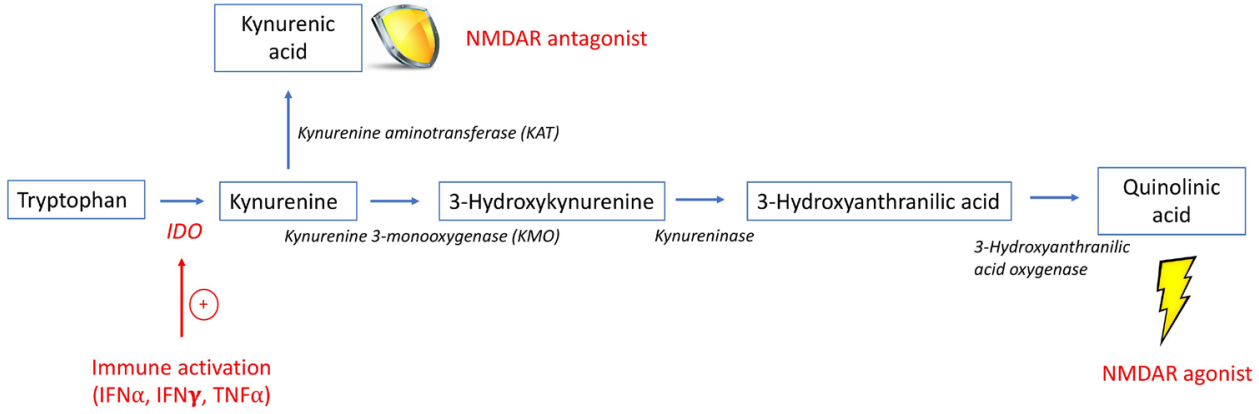

Figure 1 The kynurenine/tryptophan (KYN/TRP) pathway. This is a simplified schematic of the KYN/TRP pathway, highlighting the intermediates and enzymes involved in the production of quinolinic acid (QA) and kynurenic acid (KA). The enzyme IDO is stimulated by inflammatory cytokines, such as IFN $\alpha$, that results in the breakdown of TRP into KYN. KYN may be further metabolised by KMO ultimately to QA, an NMDAR agonist, or by KAT to KA, an NMDAR antagonist. Since the enzyme KMO has higher affinity for KYN than KAT, metabolism proceeds preferentially towards the production of QA in the setting of inflammation. ${ }^{55}$ IDO, indoleamine 2,3-dioxygenase; IFN $\alpha$, interferon-alpha; IFN $\gamma$, interferon-gamma; NMDAR, N-methyl Daspartate glutamatergic receptor; TNF $\alpha$, tumor necrosis factor-alpha.

autoantibodies, cytokines and activated microglia. ${ }^{1}$ Metabolites of the kynurenine/tryptophan (KYN/ TRP) pathway, quinolinic acid (QA) and kynurenic acid (KA), modulate neuronal function through N-methyl-Daspartate (NMDA) glutamatergic receptors (NMDARs) and are implicated in the pathogenesis of $\mathrm{CD}$ in other neurodegenerative diseases, idiopathic depression and suicidal ideation, and depression in interferon-alpha (IFN $\alpha$ )-treated patients with hepatitis C..$^{4-8}$

The KYN/TRP pathway is catalysed by the enzyme indoleamine 2,3-dioxygenase (IDO) present in immune cells in the periphery and brain. ${ }^{6}$ IFN $\alpha$, an important contributor to SLE pathogenesis, induces IDO, ${ }^{9}$ which catalyses the breakdown of TRP into KYN that is further metabolised to either QA or KA (figure 1). NMDARs on neurons are integral to learning and memory. ${ }^{10}$ As an NMDAR agonist, QA can cause glutamatergic excitotoxicity, while KA is an NMDAR antagonist that can protect neurons from excitotoxic damage. ${ }^{6}$ Animal studies demonstrate an association between peripheral IFN $\alpha$ and microglia activation, and a negative association between microglial QA and neurite outgrowth and complexity. ${ }^{11}$ Inhibitors of IDO or NMDAR prevented QA-provoked neurotoxicity in an animal model, ${ }^{12}$ suggesting that an elevated QA/KA ratio may drive NMDAR-related neurotoxicity, affecting cognitive function.

Elevated serum KYN/TRP ratios, reflective of IDO activity, are reported in subjects with $\operatorname{SLE}^{91314}$ and associated with an IFN signature. ${ }^{9}$ Metabolomic studies of peripheral blood mononuclear cells in human SLE identified elevated KYN levels as a contributor to mammalian target of rapamycin (mTOR) activation, ${ }^{15}$ associated with $\mathrm{T}$ cell dysfunction in SLE. ${ }^{16}$ Treatment with N-acetylcysteine in a small SLE clinical trial resulted in improved self-report of cognitive abilities, ${ }^{17}$ decreased KYN levels ${ }^{15}$ and decreased mTOR activity in T cells. ${ }^{18}$ Elevated cerebrospinal fluid (CSF) QA levels are reported in neuropsychiatric SLE (not limited to CD) compared with those with central nervous system (CNS) dysfunction unrelated to SLE or healthy control (HC).${ }^{19}$ While these studies suggest altered KYN/TRP pathway metabolism in SLE, perhaps driven by IFNo-induced IDO, associations between TRP metabolites and CD and depression in SLE have not been directly studied.

We hypothesised that KYN/TRP pathway activation in SLE, perhaps mediated by IFN $\alpha$, contributes to SLE-CD and depression by promoting a QA/KA imbalance. We measured serum levels of KYN, TRP, KA and QA in a cohort of subjects with SLE and HC, and concurrently assessed cognitive performance and depression.

\section{PATIENTS AND METHODS}

\section{Subject selection}

Seventy-four female subjects with SLE were recruited from Northwell Health Rheumatology Clinics. All were aged $\geq 18$ years and fulfilled SLE criteria. ${ }^{20}$ To avoid confounding influences on neuropsychological testing, key exclusion criteria for subjects with SLE included any history of neurological disease, psychiatric illness requiring medication that preceded SLE diagnosis, alcohol abuse or illicit drug use, acute infection, and current narcotic or psychiatric medication use. Patients with psychiatric illnesses diagnosed after SLE onset were allowed as long as they were not currently taking antidepressant or antipsychotic medications. Seventy-four female HC subjects, comparable with SLE subjects in age, ethnicity and race, were recruited. The HC subjects had no history of chronic medical or psychiatric illnesses and were on no medications other than oral contraception.

\section{Patient and public involvement}

Patients were not directly involved in the design, recruitment or conduct of the study.

\section{Clinical, neuropsychological and behavioural assessments}

All clinical and neuropsychological assessments, and metabolite measurements were completed within a 10-day period. For all subjects, cognition was assessed using the Automated Neuropsychological Assessment Metrics (ANAM) ${ }^{22}$ computerised test battery (selected 
tests and associated cognitive domains detailed in table 2) and a $2 \times 2$ array to assess object recognition and spatial memory. ${ }^{23}$ Throughput scores (accuracy $\div$ reaction time) were the primary measures of cognitive efficiency. Depression was evaluated using the Beck Depression Inventory (BDI; scores range from 0 to 63 , scores $>13$ indicate clinically significant depression). Additional assessments included the Numerical Rating Scale (NRS) for pain (0-10; '0' represents no pain, '10' represents worst pain imaginable) and ANAM mood subtests for fatigue, sleepiness, anger, happiness, restlessness, vigour and anxiety. For subjects with SLE, disease activity was assessed with the SELENA-SLEDAI ${ }^{24}$ and Physician's Global Assessment (PGA) ${ }^{25}$ Accrued damage since SLE diagnosis was measured with the SLICC Damage Index (SLICC-DI) ${ }^{26}$ Testing was administered by authors EWA or JH in a quiet room to minimise distractions.

\section{Analysis of KYN/TRP pathway metabolites (KYN, KA, TRP and QA)}

Metabolite concentrations in de-identified, blinded serum samples were measured by Charles River Laboratories (www.criver.com) using high-performance liquid chromatography with tandem mass spectrometry (see online supplemental material).

\section{Statistical analyses}

Metabolite data and select cognitive outcome measures (all but Running Memory Continuous Performance Test (RMCPT) and Non-Spatial Memory (NSM); see table 2) were log-transformed to better meet normality assumptions. Differences between SLE and HC for demographic and clinical characteristics, neuropsychological and behavioural test scores, and KYN/TRP pathway metabolites were assessed using t-tests, Mann-Whitney $\mathrm{U}$ tests or $\mathrm{X}^{2}$ tests, as appropriate.

Associations between demographic and clinical variables, metabolite levels and cognitive performance were analysed with Spearman's rank-order correlation, Mann-Whitney U test, Kruskal-Wallis test, $\mathrm{X}^{2}$ test or Fisher's exact test, as appropriate. Among subjects with SLE, multivariable linear regression modelling was performed to evaluate associations between individual neuropsychological test performance and clinical variables and metabolite levels. Multivariable modelling for the outcome of depression was also performed in subjects with SLE using ordinal multinomial regression methods whereby BDI was assessed as a 3-level outcome (none $(\mathrm{BDI}=0)$, minimal $(>0-\leq 13)$ and clinically significant depression $(>13)$ ).

The following variables were considered for inclusion in the multivariable models: age; ethnicity; race (black, white, other); computer experience; education years; tobacco use (ever); marijuana use (ever); history of hypertension; psychiatric illness after SLE diagnosis requiring medication prior to recruitment for this study; history of peripheral thrombosis; white blood cell count (WBC; as a continuous variable and categorised as $<3.0 \times 10^{9} / \mathrm{L}$ or $\geq 3.0 \times 10^{9} / \mathrm{L}$ ) ; haemoglobin; platelet count; BDI (categorised as 0 ; $>0-\leq 13$; $>13$ or $\leq 13$; >13) and NRS scores; ANAM mood subtest scores for fatigue, sleepiness, anger, anxiety, restlessness, happiness and vigour; serum levels of QA, KA, TRP, KYN, QA/KA and KYN/TRP; disease duration; history of lupus nephritis; history of SLE-related psychosis; prednisone dose; immunosuppressant and hydroxychloroquine use; SLEDAI and clinical SLEDAI (all descriptors except anti-double-stranded DNA (anti-dsDNA) antibodies and complement) scores; PGA; SLICC-DI; anti-dsDNA; C3, C4; urinary protein/ creatinine ratio; blood urea nitrogen; creatinine; anti-Ro and anti-La $( \pm)$.

Variables on univariate screen with $\mathrm{p}<0.1$ were considered for inclusion in each multivariable regression model. Due to skewness or lack of variability in the data, some variables were log-transformed (WBC and platelet counts) or categorised (WBC counts, BDI scores, NRS scores, all ANAM mood subtest scores), as appropriate. Multicollinearity was assessed and addressed, as appropriate. Models were created using a backward elimination strategy. The final model was chosen based on the lowest Akaike Information Criterion (AIC). If competing with a more parsimonious model, and the decrease in AIC was marginal, the more parsimonious model was selected. Analyses were conducted using SAS V.9.4 (SAS Institute). All tests of significance were two-sided, and the significance level was set at alpha $=0.05$.

\section{RESULTS}

\section{Subject characteristics}

Few significant differences were identified between subjects with SLE and HC subjects (table 1). Compared with HC subjects, a higher percentage of subjects with SLE were unemployed, had a self-reported history of $\mathrm{CD}$ and had less computer experience (table 1). In addition, a higher percentage of subjects with SLE had low WBC and haemoglobin levels, and high platelet counts. Scores on the SLEDAI ranged from 0 to 29 and current prednisone doses ranged from 0 to $75 \mathrm{mg}$ daily (table 1), indicating a broad range of disease activity.

\section{Cognitive and behavioural testing in SLE compared with HC}

Compared with HC, subjects with SLE performed significantly worse on four of five ANAM cognitive tests and the $2 \times 2$ array (table $2, \mathrm{p}<0.03$ ). Most subjects with SLE scored below the HC mean for each test: $89 \%$ scored below the HC mean in RMCPT, 84\% scored below in Match to Sample (MTS), 78\% in Spatial Memory Test (SM), 77\% in NSM, 70\% in Matching Grids (MG) and 57\% in Simple Reaction Time. Poor task performance (measured by throughput scores: accuracy $\div$ reaction time) was due to both worse accuracy and slower reaction time in MTS and NSM, while it was due to slower reaction time (with comparable accuracy) in MG, RMCPT and SM. Subjects 
Table 1 Demographics and characteristics of subjects with SLE and healthy controls (HC)

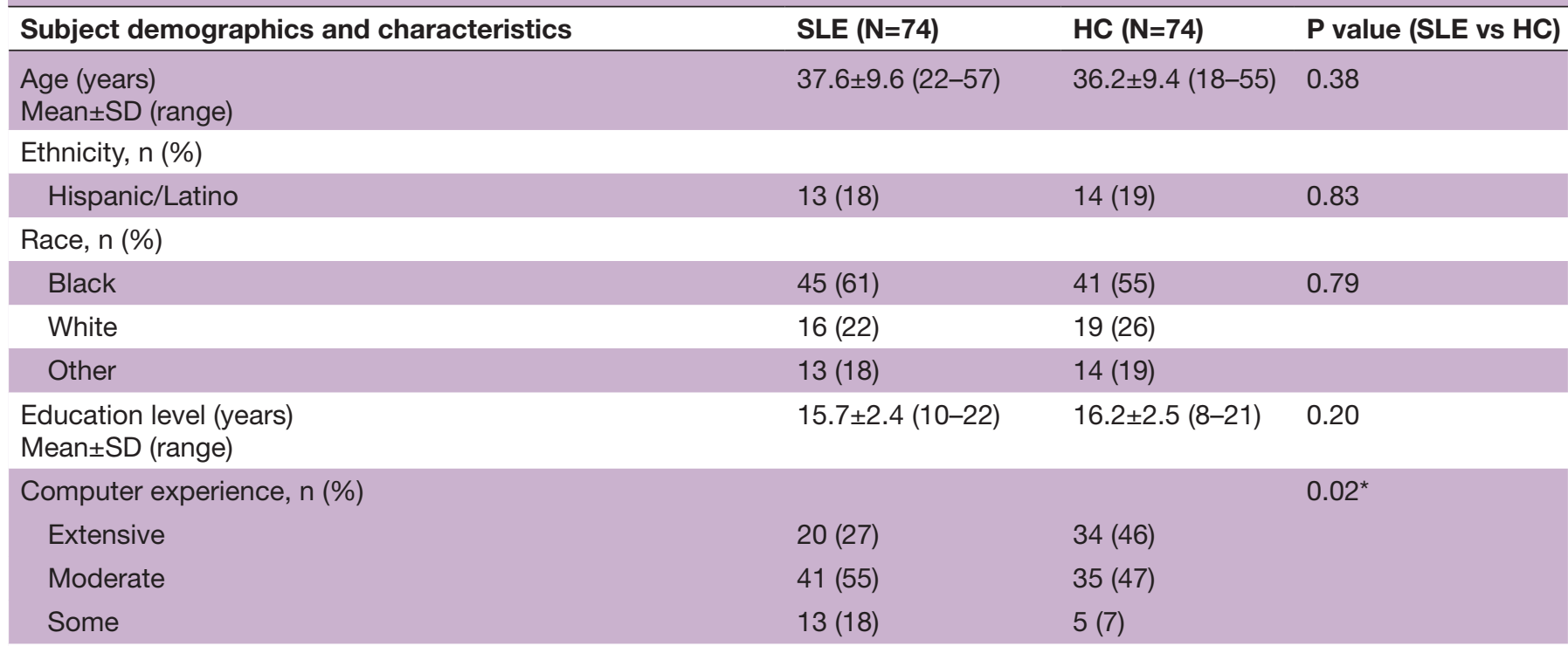

Employment status, $\mathrm{n}(\%)$

\begin{tabular}{|c|c|c|c|}
\hline Unemployed & $23(31)$ & $7(10)$ & $<0.01$ \\
\hline Self-reported cognitive dysfunction, n (\%) & $33(45)$ & $2(3)$ & $<0.01$ \\
\hline $\begin{array}{l}\text { Disease duration } \dagger \\
\text { Mean } \pm \text { SD (range) }\end{array}$ & $12.3 \pm 8.4(1-38)$ & - & - \\
\hline $\begin{array}{l}\text { SELENA-SLEDAI score } \\
\text { Mean } \pm \text { SD (range) }\end{array}$ & $5.4 \pm 5.0(0-29)$ & - & - \\
\hline $\begin{array}{l}\text { Prednisone dose, mg/day } \\
\text { Median (Q1-Q3) (range) }\end{array}$ & $2.8(0.0-10.0)(0-75)$ & - & - \\
\hline Current hydroxychloroquine use, n (\%) & $55(74)$ & - & - \\
\hline Current immunosuppressant use, n (\%) & $36(49)$ & - & - \\
\hline Anti-dsDNA positive ( $\geq 30 \mathrm{IU} / \mathrm{mL}), \mathrm{n}(\%)$ & $52(70)$ & - & - \\
\hline C3 low (<81 mg/dL), n (\%) & $29(39)$ & - & - \\
\hline $\begin{array}{l}\text { Haemoglobin } \\
n(\%) \text { less than the lower limit of the normal range }(115 \mathrm{~g} / \mathrm{L})\end{array}$ & $39(53)$ & $9(12)$ & $<0.01$ \\
\hline $\begin{array}{l}\text { Platelet count } \neq \\
n(\%) \text { greater than the upper limit of the normal range }(400 x \\
10 \%)\end{array}$ & $11(15)$ & $2(3)$ & 0.02 \\
\hline $\begin{array}{l}\text { Protein/creatinine ratio } \\
\mathrm{n}(\%) \text { with a ratio }>0.5\end{array}$ & $11(15)$ & - & - \\
\hline
\end{tabular}

All data were collected at the time of evaluation, and are reported as either a mean or median along with an SD or IQR, or as a frequency (\%). ${ }^{*}$ Extensive and some $(\mathrm{p}=0.009)$.

†Disease duration, $\mathrm{N}=74$ : $\leq 2$ years $(\mathrm{N}=8$, mean $\pm \mathrm{SD}=1.8 \pm 0.5)$; disease duration $>2$ and $<10$ years $(\mathrm{N}=24,5.8 \pm 1.9)$; disease duration $\geq 10$ years $(\mathrm{N}=42,18.0 \pm 6.7)$.

$\ddagger$ Platelet count, SLE $(\mathrm{n}=74):<150 \mathrm{~K} / \mu \mathrm{L}(\mathrm{N}=2$, mean $\pm \mathrm{SD}=140.5 \pm 2.1) ; \geq 150$ and $\leq 400(\mathrm{~N}=61,255.5 \pm 59.5) ;>400(\mathrm{~N}=11,483.2 \pm 87.3) ; \mathrm{HC}$ $(\mathrm{N}=74):<150 \mathrm{~K} / \mu \mathrm{L}(\mathrm{N}=0) ; \geq 150$ and $\leq 400(\mathrm{~N}=72,266.1 \pm 58.7) ;>400(\mathrm{~N}=2,435.0 \pm 32.5)$.

dsDNA, double-stranded DNA; HC, healthy controls.

with SLE also had higher scores for depression, pain, fatigue, sleepiness, anger and anxiety (table 2, $\mathrm{p}<0.01$ ). Among the subjects with SLE, cognitive task performance and depression were not affected by employment status (data not shown).
KYN/TRP pathway metabolites and associations with cognitive testing, depression

Subjects with SLE had higher KYN/TRP ratios (0.043 $\mu \mathrm{M}$ (0.033-0.060); median (Q1-Q3)) compared with HC $(0.032(0.025-0.037)) \quad(\mathrm{p}<0.0001)$. Similarly, higher 
Table 2 Neuropsychological and behavioural test performance in subjects with SLE compared with healthy controls (HC)

\begin{tabular}{|c|c|c|c|}
\hline Cognitive and behavioural test performance & SLE $(N=74)$ & $\mathrm{HC}(\mathrm{N}=74)$ & $\begin{array}{l}\text { P value } \\
\text { (SLE vs HC) }\end{array}$ \\
\hline \multicolumn{4}{|l|}{$\begin{array}{l}\text { Automated Neuropsychological Assessment Metrics } \\
\text { Mean (SD) }\end{array}$} \\
\hline $\begin{array}{l}\text { Simple Reaction Time } \\
\text { visuomotor processing speed, simple motor speed, attention }\end{array}$ & $363.8(88.0)$ & $321.1(65.4)$ & $<0.01$ \\
\hline $\begin{array}{l}\text { Matching Grids } \\
\text { visuospatial processing }\end{array}$ & $29.6(9.9)$ & $34.0(11.8)$ & 0.02 \\
\hline $\begin{array}{l}\text { Match to Sample } \\
\text { working memory, visuospatial processing }\end{array}$ & $25.5(8.6)$ & $31.8(12.0)$ & $<0.01$ \\
\hline $\begin{array}{l}\text { Running Memory Continuous Performance Task } \\
\text { vigilance, sustained attention, working memory }\end{array}$ & $73.4(14.7)$ & $84.0(15.6)$ & $<0.01$ \\
\hline $\begin{array}{l}\text { Spatial Processing Simultaneous } \\
\text { mental rotation, visuospatial skills }\end{array}$ & $24.0(7.0)$ & $26.3(8.8)$ & 0.26 \\
\hline \multicolumn{4}{|l|}{$\begin{array}{l}2 \times 2 \text { array } \\
\text { Mean (SD) }\end{array}$} \\
\hline $\begin{array}{l}\text { Non-Spatial Memory Test } \\
\text { working memory }\end{array}$ & $1.8(0.6)$ & $2.1(0.7)$ & $<0.01$ \\
\hline $\begin{array}{l}\text { Spatial Memory Test } \\
\text { spatial memory }\end{array}$ & $3.6(1.1)$ & $4.3(1.6)$ & 0.01 \\
\hline $\begin{array}{l}\text { Beck Depression Index score, range: 0-63 } \\
\text { Median (Q1-Q3) (range) }\end{array}$ & $\begin{array}{l}9.0(2.0-14.3) \\
(0-44)\end{array}$ & $\begin{array}{l}1.5(0.0- \\
3.0)(0-34)\end{array}$ & $<0.01$ \\
\hline \# BDI scores >13, n (\%) & $19(26)$ & $1(1)$ & $<0.01$ \\
\hline $\begin{array}{l}\text { Numerical Rating Scale for pain, range: } 0-10 \\
n(\%) \text { of subjects with score }>0\end{array}$ & $43(58)$ & $15(20)$ & $<0.01$ \\
\hline $\begin{array}{l}\text { ANAM fatigue score, range: } 0-6 \\
n(\%) \text { of subjects with score }>2\end{array}$ & $31(42)$ & $7(10)$ & $<0.01$ \\
\hline $\begin{array}{l}\text { ANAM sleepiness score, range: } 1-7 \\
n(\%) \text { of subjects with score }>2\end{array}$ & $22(30)$ & $7(10)$ & $<0.01$ \\
\hline $\begin{array}{l}\text { ANAM anger score, range: } 0-6 \\
n(\%) \text { of subjects with score }>0\end{array}$ & $32(43)$ & $9(12)$ & $<0.01$ \\
\hline $\begin{array}{l}\text { ANAM anxiety score, range: } 0-6 \\
n(\%) \text { of subjects with score }>0\end{array}$ & $51(69)$ & $32(43)$ & $<0.01$ \\
\hline
\end{tabular}

Neuropsychological test scores are all expressed as throughput, or efficiency, scores (accuracy $\div$ reaction time), except for Simple Reaction Time, which represents reaction time in milliseconds. All data are reported as a mean or median along with an SD or IQR, or as a frequency (\%).

ANAM, Automated Neuropsychological Assessment Metrics.

QA/KA ratios were found in SLE (18.3 nM (10.5-25.2)) compared with HC $(9.0 \mathrm{nM} \quad(6.2-12.2)) \quad(\mathrm{p}<0.0001)$ (figure 2). Among subjects with SLE, elevated QA/ KA ratios correlated with poor performance on MTS $(\mathrm{r}=-0.24, \mathrm{p}=0.04)$. Subjects with SLE with elevated QA/ KA ratios also had slightly higher odds of depression, but this did not reach significance $(\mathrm{OR}=1.0,95 \% \mathrm{CI}=1.0$ to 1.1, $\mathrm{p}=0.09)$. Among HC, QA/KA ratios did not correlate with performance on any cognitive test.

\section{Associations between SLE disease characteristics and metabolite ratios, cognitive and behavioural testing}

To ascertain potential confounding effects, associations between SLE-related variables (disease activity, duration, medication use) and metabolite levels, cognitive performance and behaviour were assessed. Since increased disease activity and active serology associates with increased IFN $\alpha$ activity, ${ }^{27}$ and potentially KYN/ TRP pathway activation, we assessed their relationship with metabolite ratios. Elevated KYN/TRP ratios did not associate with disease activity assessed with SLEDAI $(\mathrm{r}=0.00, \mathrm{p}=0.98)$ or PGA $(\mathrm{r}=0.03, \mathrm{p}=0.80)$. A positive correlation was identified between $\mathrm{QA} / \mathrm{KA}$ ratios and SLEDAI scores $(\mathrm{r}=0.24, \mathrm{p}=0.04)$; however, no correlations were found between QA/KA ratios and clinical SLEDAI, exclusive of serology $(\mathrm{r}=0.12, \mathrm{p}=0.30)$ or PGA scores $(\mathrm{r}=0.14, \mathrm{p}=0.23)$. Significantly higher $\mathrm{QA} / \mathrm{KA}$ ratios were found in those with elevated anti-dsDNA antibodies and hypocomplementemia (low $\mathrm{C} 3$ and/or low $\mathrm{C} 4)$ ( $\mathrm{N}=33$, mean $\pm \mathrm{SD}=26.2 \pm 20.8 \mathrm{nM})$ compared with those without this serological profile $(\mathrm{N}=41,16.0 \pm 8.7 \mathrm{nM})(\mathrm{p}<0.01)$.

No associations between disease activity and cognitive performance were identified except for modest positive 


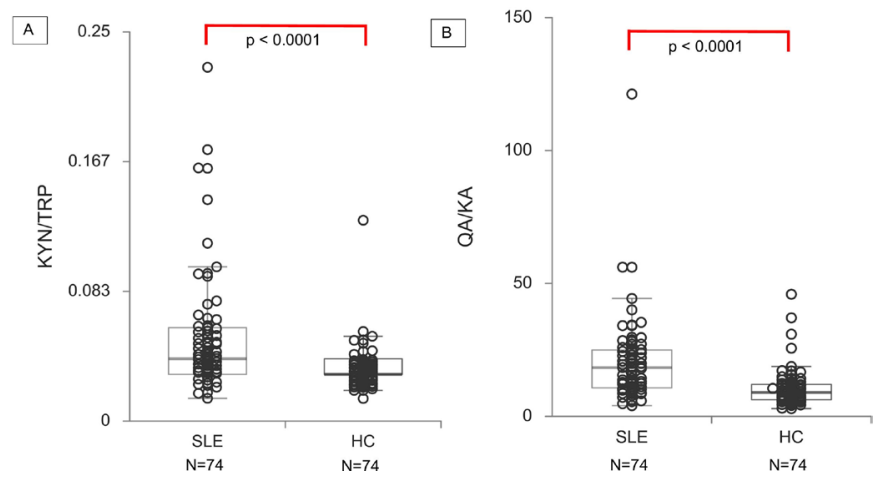

Figure $2 \mathrm{KYN} / \mathrm{TRP}$ pathway metabolite ratios in subjects with SLE compared with healthy controls (HC). Serum metabolite ratios measured by HPLC-MS in 74 subjects with SLE and $74 \mathrm{HC}$. Subjects with SLE demonstrate a significantly higher $\mathrm{KYN} / \mathrm{TRP}$ ratio $(\mathrm{A})$ and $\mathrm{QA} / \mathrm{KA}$ ratio $(B)$ than $\mathrm{HC}$. HPLC-MS, high-performance liquid chromatography-mass spectrometry; KA, kynurenic acid; $\mathrm{KYN}$, kynurenine; QA, quinolinic acid; TRP, tryptophan.

associations between MTS scores and SLEDAI $(r=0.24$, $\mathrm{p}=0.04)$ and PGA $(\mathrm{r}=0.25, \mathrm{p}=0.03)$ scores. Cognitive performance also did not associate with prednisone dose, immunosuppressant use or depression scores, but did associate with disease duration. Subjects with SLE with disease duration $\geq 10$ years $(\mathrm{N}=42$, mean $\pm \mathrm{SD}=18.0 \pm 6$.7 years) compared with those with duration $<10$ years $(\mathrm{N}=32,4.8 \pm 2.4$ years $)$ demonstrated significantly worse MTS scores $(23.5 \pm 7.3$ vs $28.0 \pm 9.6, p=0.04)$. There were no differences in KYN/TRP or QA/KA ratios between subjects with SLE with disease duration $\geq 10$ years and those $<10$ years.

Significant positive associations were found between disease activity and depression scores (SLEDAI $(r=0.40$, $\mathrm{p}<0.01)$ and PGA $(\mathrm{r}=0.41, \mathrm{p}<0.01)$ ), pain (SLEDAI $(\mathrm{r}=0.34, \mathrm{p}=<0.01)$ and PGA $(\mathrm{r}=0.36, \mathrm{p}<0.01))$, sleepiness (SLEDAI ( $\mathrm{r}=0.25, \mathrm{p}=0.04)$ and PGA $(\mathrm{r}=0.26, \mathrm{p}=0.03)$ ), fatigue (SLEDAI $(\mathrm{r}=0.24, \mathrm{p}=0.04)$ and PGA $(\mathrm{r}=0.27$, $\mathrm{p}=0.02)$ ) and anxiety (SLEDAI $(\mathrm{r}=0.35, \mathrm{p}<0.01)$ and PGA $(\mathrm{r}=0.38, \mathrm{p}<0.01))$. There was no association between anger scores and disease activity.

\section{Multivariable modelling for cognitive dysfunction and depression}

Multivariable model building included the following variables that associated with poor cognitive performance in subjects with SLE (table 3): increased age, history of psychiatric illness after SLE diagnosis requiring medication prior to the current study, increased platelet count, history of peripheral thrombosis, history of hypertension, anxiety, decreased computer experience and an elevated $\mathrm{QA} / \mathrm{KA}$ ratio. While clinically significant depression was more likely in subjects with higher disease activity and an elevated QA/KA ratio, the final, best-fit model for predicting depression included anxiety (associated with worse depression scores, $\mathrm{p}<0.01$ ) and happiness (associated with better scores, $\mathrm{p}<0.05$ ).

\section{DISCUSSION}

This is the first report of an association between an imbalanced ratio of the KYN/TRP pathway metabolites, QA/ $\mathrm{KA}$, and poor performance on a task of working memory and visuospatial processing in SLE, suggesting a potential role for this pathway in SLE-CD. In this cohort, subjects with SLE demonstrated impaired cognitive performance compared with $\mathrm{HC}$, unrelated to potentially confounding influences from disease activity, antiphospholipid syndrome (APS), medication use, educational level, employment status, fatigue, sleepiness and pain. Subjects with SLE demonstrated elevated serum KYN/ TRP ratios, suggesting IDO activation consistent with prior studies, ${ }^{9}{ }^{1314}$ and elevated QA/KA.

Increased QA/KA ratios selectively associated with poor MTS task performance. The MTS test requires a subject to select which of two checkerboard matrix designs matches the one presented $5 \mathrm{~s}$ earlier and is a test of working memory and visuospatial processing. Poor MTS performance was unique among the other ANAM tasks in that it was due to both poor accuracy and slower reaction time, rather than slower reaction time alone, perhaps representing more severe impairment. Overall, subjects with SLE performed worse than HC on every task involving working memory, supporting findings by our group and others of poor working memory performance in SLE. ${ }^{23} 28$ These data suggest that elevated QA/ KA ratios may contribute to a pathological mechanism for SLE-mediated working memory impairment. Moreover, elevated QA/KA ratios may be a therapeutic target, which is indirectly supported by a study demonstrating improved cognition in patients with SLE treated with N-acetylcysteine ${ }^{17}$ which prevented $\mathrm{KYN}$ accumulation. ${ }^{15}$

The prefrontal cortex (PFC) governs aspects of working memory and visuospatial processing ${ }^{29} 30$ and is part of a functional network engaged during MTS task performance. ${ }^{31}$ We previously demonstrated correlations between increased disease duration and diminished PFC activation on functional MRI and impaired performance on a working memory task. ${ }^{2}$ Similarly, we demonstrated decreased PFC metabolism in subjects with SLE that correlated with longer disease duration ${ }^{23} 32$ and decreased PFC volume is associated with elevated QA/ KA ratios in depressed subjects without SLE. ${ }^{7}$ Combined with our findings of associations among elevated QA/ $\mathrm{KA}$, poor working memory task performance and longer disease duration, these studies suggest a potential role for accumulated exposure to elevated QA/KA levels in PFC damage and working memory deficits. In the absence of a longitudinal study, this is a cautious interpretation as age and other factors may be important confounders.

Potential biomarkers for SLE-CD include serum levels of antibodies to NMDAR, ribosomal $\mathrm{P}$ protein/neuronal surface $\mathrm{P}$ antigen and phospholipid. ${ }^{133}$ Despite specific pathogenic mechanisms that contribute to neuron death or functional impairment, and associations with CD in animal models, they have not proven to be sensitive or specific for human SLE-CD. ${ }^{133}$ Advanced neuroimaging 


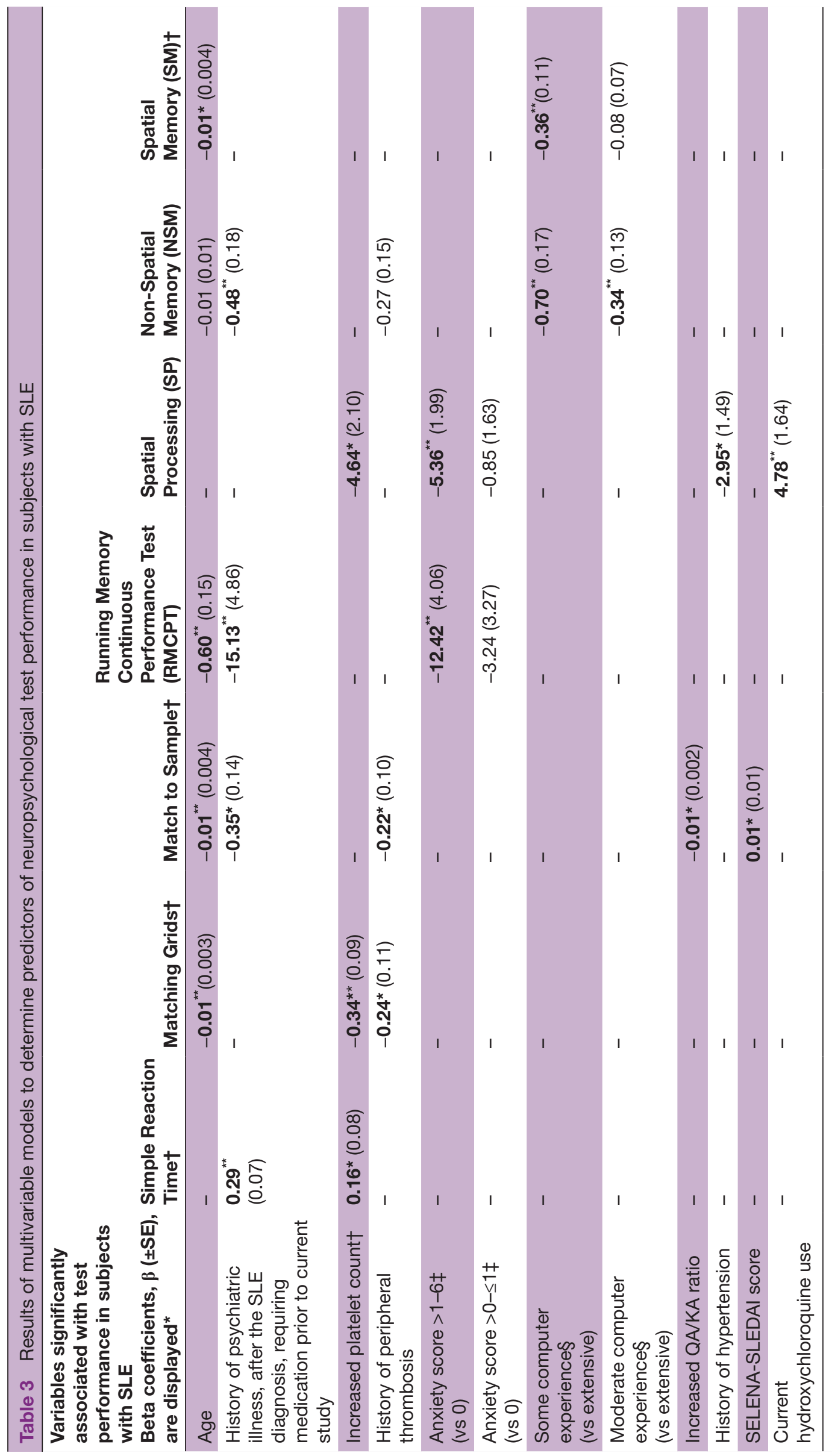

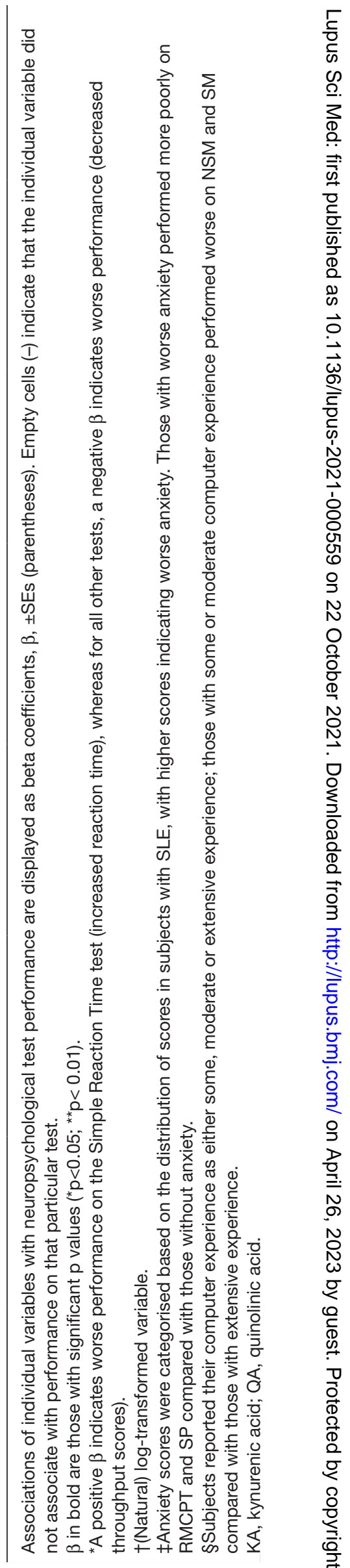


techniques are beginning to demonstrate abnormal structural or functional patterns that associate with SLE-CD. ${ }^{34}$ The potential contribution of KYN/TRP pathway activation and an elevated QA/KA ratio to SLE-CD is important because this pathway may represent a modifiable therapeutic target.

Disease activity is an important potential confounder in measurement of cognitive ability and associates with CD in some studies, while not in others. ${ }^{1}$ In our cohort, increased disease activity did not associate with poor cognitive performance. In contrast, a modest positive correlation was found between SLEDAI scores and MTS performance, independent of medication effects. However, anti-dsDNA antibodies and hypocomplementemia associated with elevated $\mathrm{QA} / \mathrm{KA}$ ratios. This is relevant in light of our hypothesis that IDO stimulation by IFN $\alpha$ contributes to increased TRP metabolism and a QA/KA imbalance, as active serology associates with high IFN $\alpha$ activity in SLE. ${ }^{27}$ Further direct study of the relationship between IFN $\alpha$ and the pathway is underway. Another potential confounder in measurement of cognitive ability is APS, mediated by potential effects on CNS microvasculature. However, only one subject in this cohort had a history of APS with no known CNS disturbance, and only 5 of 74 subjects $(7 \%)$ had elevated anticardiolipin IgG, IgM or IgA antibodies. These subjects did not perform significantly worse on any cognitive test (data not shown) than the 69 subjects who were negative for anticardiolipin IgG, IgM and IgA antibodies.

The multivariate analyses yielded increased platelets and a history of peripheral thrombosis as additional unexpected predictors of poor cognitive performance in SLE. Thrombocytosis, with ${ }^{35}$ or without ${ }^{37}$ thrombosis, has been associated with $\mathrm{CD}$ in myeloproliferative disorders and chronic inflammatory diseases, including SLE and other neurodegenerative conditions. ${ }^{36} 3839$ Our study excluded subjects with known CNS vascular disease; however, standard neuroimaging may fail to detect microinfarction. ${ }^{40}{ }^{41}$ Platelet activation is a contributor to cardiovascular, renal and thrombotic disease in SLE due to proinflammatory mediators such as type I IFN. ${ }^{36}$ Therefore, our observation that increased platelets associated with poor cognitive performance deserves further study.

Depression is highly prevalent in SLE but attribution to SLE-mediated mechanisms apart from other factors is compromised by limited studies of biological mechanisms in humans. In patients without SLE, elevated serum QA/ $\mathrm{KA}$ associates with severe depression requiring electroconvulsive therapy ${ }^{42}$ and elevated CSF QA levels were found in suicidal patients and in brains of suicide completers. ${ }^{7}$ Notably, only three subjects with SLE in our cohort had severe depression (BDI score $>28$ ). These subjects had higher mean $\mathrm{QA} / \mathrm{KA}$ ratios $(\mathrm{N}=3$, mean $\pm \mathrm{SD}=29.2 \pm 9.5)$ than subjects without severe depression $(\mathrm{BDI} \leq 28, \mathrm{~N}=71$, $20.2 \pm 16.2)$, but this did not reach significance $(p<0.1)$. The lack of a significant association between $\mathrm{QA} / \mathrm{KA}$ ratios and depression may be related to the small number of severely depressed subjects with SLE in our cohort.

There are several potential limitations to this study. First, in the absence of preliminary data on associations between metabolite ratios and cognitive performance or depression, the study was powered to detect differences in metabolite ratios between SLE and HC. It is possible that elevated $\mathrm{QA} / \mathrm{KA}$ ratios may associate with poor performance on additional tests or depression in a larger cohort. Second, CSF metabolite levels would provide direct evidence of KYN/TRP metabolites in the brain. KYN and TRP cross the blood-brain barrier more easily than QA and $\mathrm{KA} ;{ }^{44}$ however, both peripheral KYN and QA contribute to brain levels of these metabolites under systemic inflammatory conditions, ${ }^{45}$ and several human studies report positive correlations between blood and CSF levels of KYN, TRP ${ }^{46-48}$ and QA. ${ }^{49-51}$ Our study was cross-sectional, and chronic KYN/TRP pathway activation may negatively impact SLE-CD longitudinally, suggested by associations among disease duration, working memory and QA/KA levels. Studies are underway to determine longitudinal changes in KYN/TRP pathway metabolites and associations between IFN $\alpha$ and metabolite ratios and changes in cognition and depression. Future studies may also consider comparator groups consisting of primary neuropsychiatric or other autoimmune diseases. While we attempted to control for drugs that affect IDO, exclusion of all potentially interfering drugs, such as glucocorticoids, would have negatively impacted the study's feasibility and generalisability. However, most of these drugs inhibit rather than stimulate IDO ${ }^{52}{ }^{53}$ which would have reduced our chance of identifying elevated metabolite ratios in SLE. Lastly, dietary TRP intake was not assessed, although dietary impact on KYN/TRP pathway metabolism is uncertain. ${ }^{54}$

Our carefully conducted assessments of subjects with SLE without confounding CNS disease identified a novel association between elevated QA/KA ratios and poor cognitive performance. The established neurotoxicity of QA, coupled with a plausible pathophysiological mechanism for a QA/KA imbalance in SLE by IFN $\alpha$ stimulation of IDO, support additional study of the KYN/TRP pathway as a potential biomarker or therapeutic target for SLE-CD. The therapeutic potential of the pathway is highlighted by the development of non-immunosuppressive KA analogues or KYN/TRP pathway enzyme inhibitors ${ }^{8}$ to shift QA/KA ratios towards a neuroprotective balance.

\section{Author affiliations}

${ }^{1}$ Institute of Molecular Medicine, Feinstein Institutes for Medical Research,

Manhasset, New York, USA

${ }^{2}$ Charles River Laboratories, South San Francisco, California, USA

Acknowledgements The authors would like to express their gratitude towards the patients and healthy individuals who participated in this study, and for the support of the research coordinators and nurses at the Feinstein Institutes.

Contributors Study design-EWA, MM, CA and BD. Data collection-EWA, MM, $\mathrm{CA}, \mathrm{RAF}, \mathrm{JR}$ and JH. Data analysis-EWA, JF, MM, CA, BD, BV and JR. Interpretation of findings—EWA, JF, MM, CA, BD, BV and RAF. Preparation of manuscript-EWA, 
JF, MM, CA, BD, BV, RAF and JR. Guarantor-EWA. All authors read and approved the final manuscript.

Funding This work was supported by a grant from the Lupus Foundation of America (Gary S Gilkeson Career Development Award, G-1904-00773, PI: EWA; October 2019-September 2021).

Competing interests None declared.

Patient consent for publication Not required.

Ethics approval The research complied with the Helsinki Declaration and was approved by Northwell Health's Institutional Review Board. Informed consent was obtained prior to study procedures.

Provenance and peer review Not commissioned; externally peer reviewed. Data availability statement All data relevant to the study are included in the article or uploaded as supplemental information.

Supplemental material This content has been supplied by the author(s). It has not been vetted by BMJ Publishing Group Limited (BMJ) and may not have been peer-reviewed. Any opinions or recommendations discussed are solely those of the author(s) and are not endorsed by BMJ. BMJ disclaims all liability and responsibility arising from any reliance placed on the content. Where the content includes any translated material, BMJ does not warrant the accuracy and reliability of the translations (including but not limited to local regulations, clinical guidelines, terminology, drug names and drug dosages), and is not responsible for any error and/or omissions arising from translation and adaptation or otherwise.

Open access This is an open access article distributed in accordance with the Creative Commons Attribution Non Commercial (CC BY-NC 4.0) license, which permits others to distribute, remix, adapt, build upon this work non-commercially, and license their derivative works on different terms, provided the original work is properly cited, appropriate credit is given, any changes made indicated, and the use is non-commercial. See: http://creativecommons.org/licenses/by-nc/4.0/.

\section{ORCID iDs}

Erik W Anderson http://orcid.org/0000-0003-3154-9648

Richard A Furie http://orcid.org/0000-0001-6712-1585

Cynthia Aranow http://orcid.org/0000-0001-9299-0053

Betty Diamond http://orcid.org/0000-0002-3250-3804

\section{REFERENCES}

1 Kello N, Anderson E, Diamond B. Cognitive dysfunction in systemic lupus erythematosus: a case for initiating trials. Arthritis Rheumatol 2019;71:1413-25.

2 Mackay M, Bussa MP, Aranow C, et al. Differences in regional brain activation patterns assessed by functional magnetic resonance imaging in patients with systemic lupus erythematosus stratified by disease duration. Mol Med 2011;17:1349-56.

3 Ramage AE, Fox PT, Brey RL, et al. Neuroimaging evidence of white matter inflammation in newly diagnosed systemic lupus erythematosus. Arthritis Rheum 2011;63:3048-57.

4 Gulaj E, Pawlak K, Bien B, et al. Kynurenine and its metabolites in Alzheimer's disease patients. Adv Med Sci 2010;55:204-11.

5 Giil LM, Midttun Øivind, Refsum H, et al. Kynurenine pathway metabolites in Alzheimer's disease. J Alzheimers Dis 2017;60:495-504.

6 Schwarcz R, Stone TW. The kynurenine pathway and the brain: challenges, controversies and promises. Neuropharmacology 2017;112:237-47.

7 Savitz J. Role of kynurenine metabolism pathway activation in major depressive disorders. Curr Top Behav Neurosci 2017;31:249-67.

8 Stone TW, Darlington LG. The kynurenine pathway as a therapeutic target in cognitive and neurodegenerative disorders. Br J Pharmacol 2013;169:1211-27.

9 Lood C, Tydén H, Gullstrand B, et al. Type I interferon-mediated skewing of the serotonin synthesis is associated with severe disease in systemic lupus erythematosus. PLoS One 2015;10:e0125109.

10 Latif-Hernandez A, Shah D, Ahmed T, et al. Quinolinic acid injection in mouse medial prefrontal cortex affects reversal learning abilities, cortical connectivity and hippocampal synaptic plasticity. Sci Rep 2016;6:36489.

11 Aw E, Zhang Y, Carroll M. Microglial responses to peripheral type 1 interferon. J Neuroinflammation 2020;17:340.

12 O'Farrell K, Fagan E, Connor TJ, et al. Inhibition of the kynurenine pathway protects against reactive microglial-associated reductions in the complexity of primary cortical neurons. Eur J Pharmacol 2017;810:163-73.
13 Åkesson K, Pettersson S, Ståhl S, et al. Kynurenine pathway is altered in patients with SLE and associated with severe fatigue. Lupus Sci Med 2018;5:e000254.

14 Widner B, Sepp N, Kowald E, et al. Degradation of tryptophan in patients with systemic lupus erythematosus. Adv Exp Med Biol 1999;467:571-7.

15 Perl A, Hanczko R, Lai Z-W, et al. Comprehensive metabolome analyses reveal $N$-acetylcysteine-responsive accumulation of kynurenine in systemic lupus erythematosus: implications for activation of the mechanistic target of rapamycin. Metabolomics 2015;11:1157-74.

16 Fernandez D, Perl A. mTOR signaling: a central pathway to pathogenesis in systemic lupus erythematosus? Discov Med 2010;9:173-8.

17 Garcia RJ, Francis L, Dawood M, et al. Attention deficit and hyperactivity disorder scores are elevated and respond to $\mathrm{N}$-acetylcysteine treatment in patients with systemic lupus erythematosus. Arthritis Rheum 2013;65:1313-8.

18 Lai Z-W, Hanczko R, Bonilla E, et al. N-Acetylcysteine reduces disease activity by blocking mammalian target of rapamycin in T cells from systemic lupus erythematosus patients: a randomized, double-blind, placebo-controlled trial. Arthritis Rheum 2012;64:2937-46.

19 Vogelgesang SA, Heyes MP, West SG, et al. Quinolinic acid in patients with systemic lupus erythematosus and neuropsychiatric manifestations. J Rheumatol 1996;23:850-5.

20 Hochberg MC. Updating the American College of rheumatology revised criteria for the classification of systemic lupus erythematosus. Arthritis Rheum 1997;40:40.

21 Petri M, Orbai A-M, Alarcón GS, et al. Derivation and validation of the systemic lupus international collaborating clinics classification criteria for systemic lupus erythematosus. Arthritis Rheum 2012;64:2677-86.

22 Bleiberg J, Kane RL, Reeves DL, et al. Factor analysis of computerized and traditional tests used in mild brain injury research. Clin Neuropsychol 2000;14:287-94.

23 Mackay M, Vo A, Tang CC, et al. Metabolic and microstructural alterations in the SLE brain correlate with cognitive impairment. $\mathrm{JCl}$ Insight 2019;4. doi:10.1172/jci.insight.124002. [Epub ahead of print: 10 Jan 2019].

24 Petri M, Buyon J, Kim M. Classification and definition of major flares in SLE clinical trials. Lupus 1999;8:685-91.

25 Lam GKW, Petri M. Assessment of systemic lupus erythematosus. Clin Exp Rheumatol 2005;23:S120-32.

26 Gladman DD, Urowitz MB, Goldsmith $\mathrm{CH}$, et al. The reliability of the systemic lupus international collaborating Clinics/American College of rheumatology damage index in patients with systemic lupus erythematosus. Arthritis Rheum 1997;40:809-13.

27 Rönnblom L, Leonard D. Interferon pathway in SLE: one key to unlocking the mystery of the disease. Lupus Sci Med 2019;6:e000270.

$28 \mathrm{RC} \mathrm{H}$, Husain SF, CS H. Cognitive dysfunction in patients with systemic lupus erythematosus: the challenge in diagnosis and management. Rheumatology Practice and Research 2018;3:2059902118792434.

29 Funahashi S. Working memory in the prefrontal cortex. Brain Sci 2017;7. doi:10.3390/brainsci7050049. [Epub ahead of print: 2704 2017].

30 Kravitz DJ, Saleem KS, Baker Cl, et al. A new neural framework for visuospatial processing. Nat Rev Neurosci 2011;12:217-30.

31 Daniel TA, Katz JS, Robinson JL. Delayed match-to-sample in working memory: a BrainMap meta-analysis. Biol Psychol 2016;120:10-20.

32 Mackay M, Tang CC, Volpe BT, et al. Brain metabolism and autoantibody titres predict functional impairment in systemic lupus erythematosus. Lupus Sci Med 2015;2:e000074.

33 Seet D, Allameen NA, Tay SH, et al. Cognitive dysfunction in systemic lupus erythematosus: immunopathology, clinical manifestations, neuroimaging and management. Rheumatol Ther 2021;8:651-79.

34 Mackay M, Tang CC, Vo A. Advanced neuroimaging in neuropsychiatric systemic lupus erythematosus. Curr Opin Neurol 2020;33:353-61.

35 Vannucchi AM, Barbui T. Thrombocytosis and thrombosis. Hematology Am Soc Hematol Educ Program 2007:363-70.

36 Scherlinger M, Sisirak V, Richez C, et al. New insights on platelets and platelet-derived microparticles in systemic lupus erythematosus. Curr Rheumatol Rep 2017;19:48.

37 Horstman LL, Jy W, Ahn YS, et al. Role of platelets in neuroinflammation: a wide-angle perspective. J Neuroinflammation 2010;7:10. 
38 Kuriyama N, Mizuno T, Yasuike H, et al. CD62-mediated activation of platelets in cerebral white matter lesions in patients with cognitive decline. Arch Gerontol Geriatr 2016;62:118-24.

39 Pluta R, Ułamek-Kozioł M, Januszewski S, et al. Platelets, lymphocytes and erythrocytes from Alzheimer's disease patients: the quest for blood cell-based biomarkers. Folia Neuropathol 2018;56:14-20.

40 Katsumata Y, Harigai M, Kawaguchi Y, et al. Diagnostic reliability of magnetic resonance imaging for central nervous system syndromes in systemic lupus erythematosus: a prospective cohort study. BMC Musculoskelet Disord 2010;11:13.

41 Cohen D, Rijnink EC, Nabuurs RJA, et al. Brain histopathology in patients with systemic lupus erythematosus: identification of lesions associated with clinical neuropsychiatric lupus syndromes and the role of complement. Rheumatology 2017;56:77-86.

42 Schwieler L, Samuelsson M, Frye MA, et al. Electroconvulsive therapy suppresses the neurotoxic branch of the kynurenine pathway in treatment-resistant depressed patients. J Neuroinflammation 2016;13:51

43 Ryan KM, Allers KA, McLoughlin DM, et al. Tryptophan metabolite concentrations in depressed patients before and after electroconvulsive therapy. Brain Behav Immun 2020;83:153-62.

44 Fukui S, Schwarcz R, Rapoport SI, et al. Blood-Brain barrier transport of kynurenines: implications for brain synthesis and metabolism. J Neurochem 1991;56:2007-17.

45 Kita T, Morrison PF, Heyes MP, et al. Effects of systemic and central nervous system localized inflammation on the contributions of metabolic precursors to the L-kynurenine and quinolinic acid pools in brain. J Neurochem 2002;82:258-68.

46 Curzon G. Relationships between plasma, CSF and brain tryptophan J Neural Transm Suppl 1979:81-92.
47 Chen Y, Stankovic R, Cullen KM, et al. The kynurenine pathway and inflammation in amyotrophic lateral sclerosis. Neurotox Res 2010;18:132-42.

48 Jacobs KR, Lim CK, Blennow K, et al. Correlation between plasma and CSF concentrations of kynurenine pathway metabolites in Alzheimer's disease and relationship to amyloid- $\beta$ and tau. Neurobiol Aging 2019;80:11-20.

49 Raison CL, Dantzer R, Kelley KW, et al. CSF concentrations of brain tryptophan and kynurenines during immune stimulation with IFNalpha: relationship to CNS immune responses and depression. Mol Psychiatry 2010;15:393-403.

50 Saito K, Crowley JS, Markey SP, et al. A mechanism for increased quinolinic acid formation following acute systemic immune stimulation. J Biol Chem 1993;268:15496-503.

51 Valle M, Price RW, Nilsson A, et al. CSF quinolinic acid levels are determined by local HIV infection: cross-sectional analysis and modelling of dynamics following antiretroviral therapy. Brain 2004;127:1047-60.

52 Gostner JM, Schröcksnadel S, Becker K, et al. Antimalarial drug chloroquine counteracts activation of indoleamine $(2,3)$-dioxygenase activity in human PBMC. FEBS Open Bio 2012;2:241-5.

53 Schroecksnadel S, Sucher R, Kurz K, et al. Influence of immunosuppressive agents on tryptophan degradation and neopterin production in human peripheral blood mononuclear cells. Transpl Immunol 2011;25:119-23.

54 Richard DM, Dawes MA, Mathias CW, et al. L-Tryptophan: basic metabolic functions, behavioral research and therapeutic indications. Int J Tryptophan Res 2009;2:IJTR.S2129-60.

55 Lugo-Huitrón R, Ugalde Muñiz P, Pineda B, et al. Quinolinic acid: an endogenous neurotoxin with multiple targets. Oxid Med Cell Longev 2013;2013:1-14. 